\title{
Effects of Pioglitazone on the Electrocardiogram in the Goto-Kakizaki Type 2 Diabetic Rat Heart
}

\author{
Salem KA ${ }^{1}$, Jacobson $\mathrm{M}^{2}$, Shafiullah $\mathrm{M}^{1}, \mathrm{Oz} \mathrm{M}^{1}$, Adeghate $\mathrm{E}^{1}$ and Howarth $\mathrm{FC}^{* 1}$
}

${ }^{1}$ Departments of Pharmacology, Physiology and Anatomy, College of Medicine and Health Sciences, Al Ain, UAE University, UAE

${ }^{2}$ Electrical and Electronic Engineering Technology Department, Higher Colleges of Technology, Abu Dhabi, UAE *Corresponding author: Howarth FC, Departments of Pharmacology, Physiology and Anatomy, College of Medicine and Health Sciences, Al Ain, UAE University, UAE, Tel: 009713 7137536, E-mail: chris.howarth@ uaeu.ac.ae

Citation: Salem KA, Jacobson M, Shafiullah M, Oz M, Adeghate E, et al. (2015) Effects of Pioglitazone on the Electrocardiogram in the Goto-Kakizaki Type 2 Diabetic Rat Heart. J Clin Exp Res Cardiol 2(1): 101. doi: 10.15744/2394-6504.2.101

\section{Received Date: April 12, 2015 Accepted Date: May 18, 2015 Published Date: May 19, 2015}

\begin{abstract}
Cardiovascular complications are the major cause of morbidity and mortality in diabetic patients. Pioglitazone (PIO) is used for the treatment of type 2 diabetes mellitus and there is some evidence that it may improve ventricular function in diabetic patients. The effects of PIO on electrical conduction in the Goto-Kakizaki (GK) type 2 diabetic rat heart have been investigated. Transmitter devices were surgically implanted in GK and control rats aged 7 months. PIO at concentrations ( 2.5 to $20 \mathrm{mg} / \mathrm{kg}$ ) was added to the drinking water. Animals received each concentration of PIO for 2 weeks. Bodyweight and blood glucose were measured periodically. The electrocardiogram was recorded throughout the study. Blood glucose was higher in GK rats compared to controls and was progressively reduced in GK rats treated with increasing concentrations of PIO. A modest reduction in heart rate was associated with a significant increase in PQ interval in GK rats compared to controls. Heart rate and PQ interval were not additionally altered by PIO. QRS complex and QT interval were not altered in GK compared to control or by PIO. In conclusion daily oral doses of PIO in the range 2.5 to $20 \mathrm{mg} /$ $\mathrm{kg}$ during a period of 14 weeks appeared to have little effect on electrical conduction in GK and control rat heart.
\end{abstract}

Keywords: Electrical conduction; Goto-Kakizaki rat; Heart; In vivo biotelemetry; Type 2 diabetes mellitus

\section{Introduction}

It is well known that the prevalence of diabetes mellitus in the United Arab Emirates is among the highest in the world and that cardiovascular complications are the major cause of morbidity and mortality in diabetic patients [1]. Pioglitazone (PIO) belongs to the thiazolidinedione group of oral anti-diabetic agents, and is approved in many countries for the treatment of type 2 diabetes mellitus and can be administered in combination with metformin, sulfonylureas, exenatide, dipeptidyl peptidase 4 inhibitors or insulin. The thiazolidinediones are insulin sensitizers which act via activation of peroxisome proliferator-activated receptors (PPAR). PIO improves glycosylated hemoglobin, fasting blood glucose and $\beta$-cell function. It also increases glucose uptake in the periphery while decreasing glucose production in the liver, produces favourable effects on lipid profile and blood pressure and decreases the risk of certain cardiovascular events. PIO in combination with any insulin containing regimen slightly increases the risk of hypoglycaemic episodes and may cause weight gain and oedema due to fluid retention, and increases the risk for the development and/or exacerbation of heart failure [2,3]. Experimentally PIO has been shown to decrease fasting and postprandial insulin concentrations and lower blood pressure in spontaneously hypertensive rats [4]. PIO improves fasting and postprandial levels of insulin sensitivity, plasma glucose and lipid levels and also decreases systolic and mean arterial pressure in spontaneously obese, insulin-resistant rhesus monkey [5]. High oral doses of PIO produces cardiac hypertrophy and mild congestion of the liver and kidneys in mice [6]. There is some evidence that PIO may lead to improved ventricular diastolic function in diabetic patients $[7,8]$. In addition to an increased risk of heart attack and stroke, cardiac electrical conduction abnormalities are frequently observed in diabetic patients [9-11]. These include various arrhythmias [9-11], atrioventricular block [12], prolonged QT interval and sudden death $[10,11,13]$. Hyperglycaemia has been linked to a prolonged QT interval, and the presence of QT abnormalities are commonly used as indicators of mortality in diabetic patients [13,14]. It is also noteworthy that the incidence of bundle branch block, bradyarrhythmias and atrioventricular block are significantly higher in diabetic patients than in the general population [12]. The Goto-Kakizaki (GK) rat is a well characterized experimental model of type 2 diabetes mellitus displaying a variety of characteristics that are frequently observed in type 2 diabetic patients including elevated blood glucose, insulin resistance, and disturbances in cholesterol, triglyceride, high-density and low-density lipoproteins [15,16]. The hemodynamic function of the GK rat heart is compromised and reported defects include reduced ejection fraction, altered amplitude and prolonged time course of cardiac muscle contraction $[17,18]$. Previous studies in our laboratory have demonstrated a variety of electrical conduction distu- 
rbances in GK rat heart including reduction in heart rate and heart rate variability, prolongation of QRS complex duration and QT interval and these changes may occur from an early age [19]. To our knowledge there have been no studies to investigate the effects of PIO on electrical conduction in type 2 diabetic heart.

\section{Methods}

\section{Biotelemetry system}

Heart biopotential was monitored with a biotelemetry system (Data Sciences Int., St Paul, MN, USA) using previously described techniques $[19,20]$. The system comprised the transmitter devices (TA11CTA-F40, Data Sciences Int.), the receivers (RPC-1, Data Sciences Int.) a data exchange matrix (20CH, Data Sciences Int.) and a personnel computer for system configuration, control, acquisition, storage and analysis. The transmitter devices were surgically implanted in 7 GK and 7 Wistar male control rats, 6 months of age, under general anesthesia (sodium pentobarbitone, $45 \mathrm{mg} \mathrm{kg}^{-1}$, intraperitoneal). Transmitter devices were inserted into the peritoneal cavity with the electrodes protruding caudally (towards the tail) and arranged in Einthoven bipolar lead II configuration (right foreleg and left side of chest at the level of the last rib). Transmitters were activated by use of a permanent magnet switch. Ethical approval for the project was obtained from the College of Medicine \& Health Sciences Ethics Committee for Animal Research.

\section{Experimental protocol}

Before surgical implantation of the transmitter devices, non-fasting blood glucose and bodyweight were measured. After an overnight fast a glucose tolerance test was administered. Blood glucose was measured at time zero (fasting blood glucose) and 120 min following administration of glucose $(2 \mathrm{~g} / \mathrm{kg}$, intraperitoneal). Surgical implantation of transmitters was then performed in animals aged 7 months. Animals received standard rat chow ad libitum throughout the experiment. Data acquisition commenced one week after surgery and continued throughout the study. During the first few weeks animals received normal drinking water. PIO was dissolved in dimethyl sulfoxide (DMSO) and then added to the drinking water. During week 4 animals received Water/ DMSO (0.19\% v/v), week 5-6 Water/DMSO/PIO $2.5 \mathrm{mg}$, week 7-8 Water/ DMSO/PIO 5 mg, week 9-10 Water/DMSO/PIO 10 mg, week 11-12 Water/DMSO/PIO $20 \mathrm{mg}$ and weeks 13-14 back to Water/DMSO. Acquisition was then stopped. Bodyweight and nonfasting blood glucose was measured at the end of each dose of PIO and at the end of the experiment.

\section{Data collection}

Electrocardiogram (ECG) data was collected for 5 min per hour per animal, 24 h per day. From the collected ECG data, secondary physiological measurements were determined, including the average 5 min heart rate, PQ, QRS and QT intervals.

\section{Statistical analysis}

Unless otherwise stated, statistical comparisons were made using analysis of variance (ANOVA) with Bonferroni post hoc using IBM SPSS Statistics for Windows, Version 20.0. (Armonk, NY: IBM Corp.). P values less than 0.05 were considered significant.

\section{Results}

General characteristics of the animals: Prior to implantation of the transmitter devices the fasting blood glucose was significantly higher in GK rats $(101.3 \pm 5.6 \mathrm{mg} / \mathrm{dl}, \mathrm{n}=7)$ compared to age matched controls $(73.9 \pm 5.6 \mathrm{mg} / \mathrm{dl}, \mathrm{n}=7)$. Blood glucoseat $120 \mathrm{~min}$ after glucose injection ( $2 \mathrm{~g} / \mathrm{kg}$ bodyweight, intraperitoneal) was $283.7 \pm 24.5 \mathrm{mg} / \mathrm{dl}$ in $\mathrm{GK}$ rats compared to $91.9 \pm 4.0 \mathrm{mg} / \mathrm{dl} \mathrm{in}$ controls. Average body weight, fasting blood glucose and blood glucose at $120 \mathrm{~min}$ following glucose administration were all significantly higher in GK rats compared to controls (Table 1).

\begin{tabular}{|c|c|c|}
\hline & Control $(\mathbf{n}=7)$ & GK $(\mathbf{n}=7)$ \\
\hline Bodyweight $(\mathbf{g})$ & $345.3 \pm 10.5$ & $407.7 \pm 7.4^{*}$ \\
\hline Fasting blood glucose $(\mathbf{m g} / \mathbf{d l})$ & $73.9 \pm 5.6$ & $101.3 \pm 5.6^{*}$ \\
\hline GTT $(\mathbf{m g} / \mathbf{d l})$ at $\mathbf{1 2 0} \mathbf{~ m i n}$ & $91.9 \pm 4$ & $283.7 \pm 24.5^{*}$ \\
\hline \\
Table 1: General characteristic of the animals prior to implantation of transmitters
\end{tabular}

Bodyweights of GK rats and controls measured at different stages of the study are shown in Figure 1. Bodyweights of GK rats were significantly higher than controls at all stages of the experiment. PIO had no significant effects on bodyweight in either GK rats or controls. Non-fasting blood glucose in GK rats and controls measured at different stages of the study are shown in Figure 2. At the start of the experiment blood glucose was significantly higher in GK rats compared to controls. With increasing concentrations of PIO blood glucose in GK rats was reduced towards control values. PIO appeared to have little effect in controls.

Typical records of average heart rate are shown in Figure 3A. Heart rate was modestly lower in GK rats compared to controls (Figure 3B). PIO had no significant on heart rate in either GK rats or controls. Interestingly, the PQ interval was significantly prolonged in GK rats compared to controls before administration of PIO (Figure 4A). PIO had no significant effects on the PQ interval in either GK rats or controls. QRS complex duration was not significantly altered in GK compared to controls or by PIO (Figure 4B). QT (Figure 5A) and QT corrected for heart rate (Figure 5B) were not significantly altered in GK rats compared to controls or by PIO. 


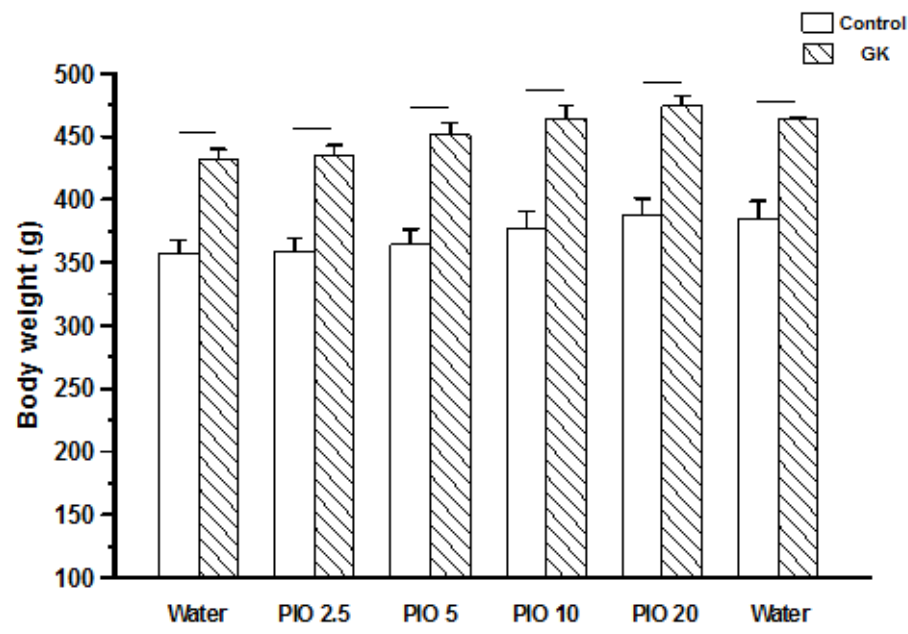

Figure 1: Effects of Pioglitazone (PIO) on bodyweight gain in GK rats and control. Data are mean \pm S.E.M., $n=7$. Numbers on the $\mathrm{X}$-axis represent the dose in $\mathrm{mg} / \mathrm{kg} /$ day. Lines above bars indicate significant differences $\mathrm{P}<0.05$

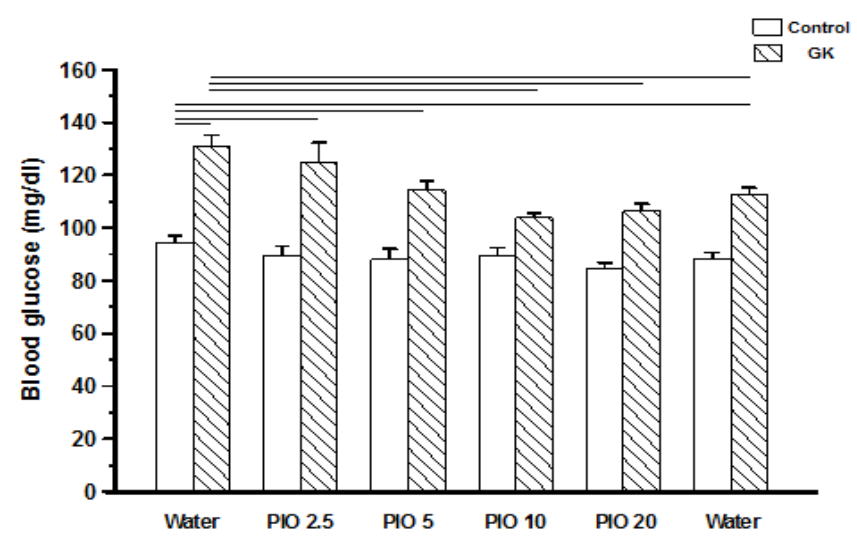

Figure 2: Effects of Pioglitazone (PIO) on non-fasting blood glucose in GK rats and control. Data are mean \pm S.E.M., $\mathrm{n}=7$. Numbers on the $\mathrm{X}$-axis represent the dose in $\mathrm{mg} / \mathrm{kg} /$ day. Lines above bars indicate significant differences $\mathrm{P}<0.05$

A

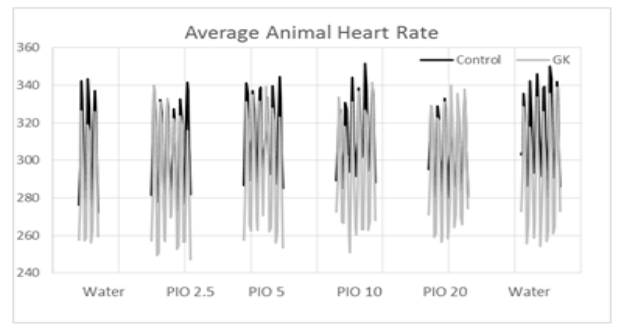

B

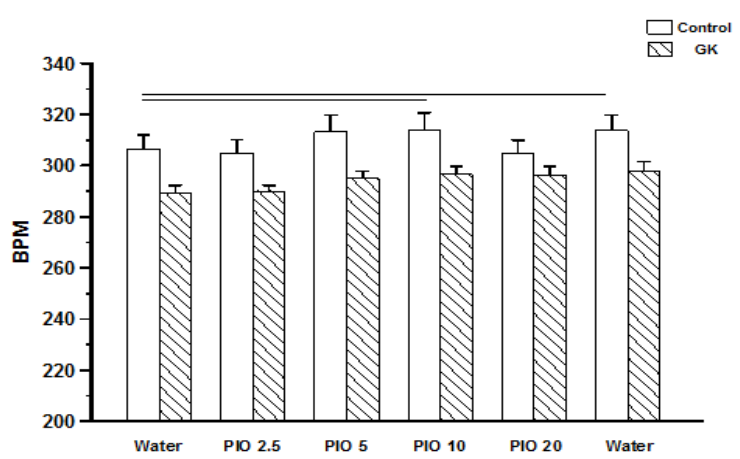

Figure 3: Effects of Pioglitazone (PIO) on heart rate in GK rats compared to controls. A: Typical recording of heart rate. B: Mean heart rate in GK rats and controls during administration of different concentrations of PIO. Data are means \pm S.E.M., $n=7$. Numbers on the X-axis represent the dose in $\mathrm{mg} / \mathrm{kg} /$ day. Lines above bars indicate significant differences $\mathrm{P}<0.05$ 


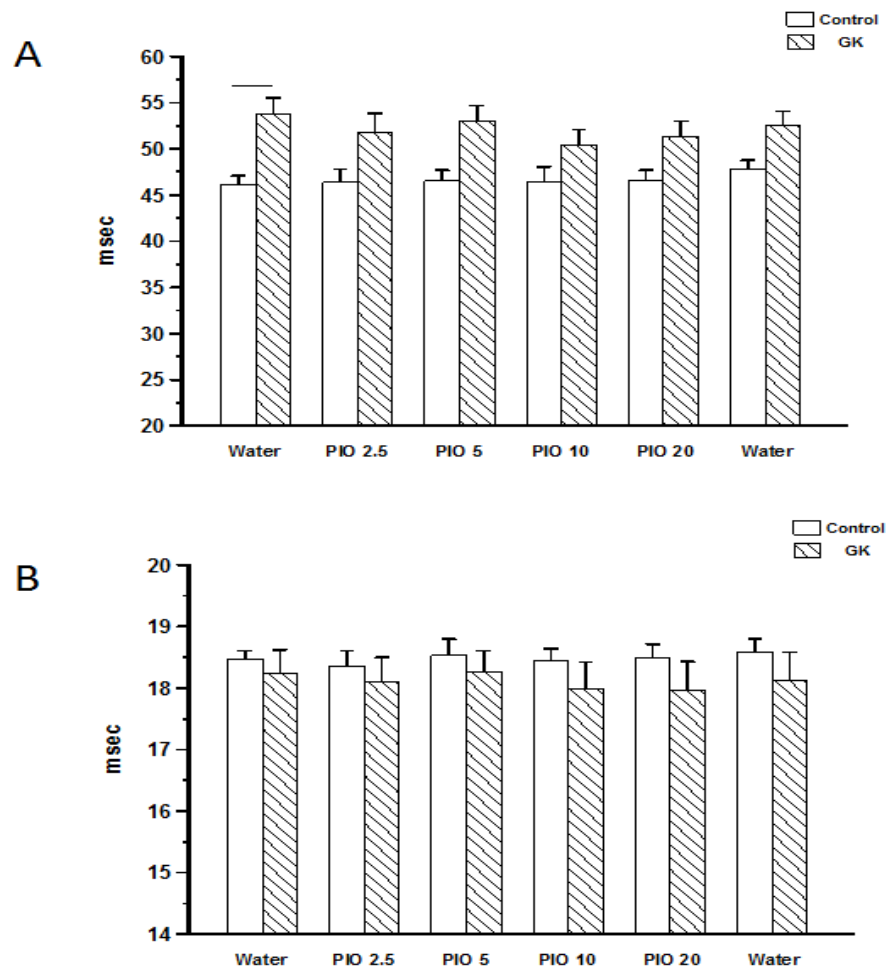

Figure 4: Effects of Pioglitazone (PIO) on PQ and QRS intervals in GK rats and controls. A: Mean PQ interval and B: Mean QRS interval in GK rats and controls during administration of different concentrations of PIO. Data are means \pm S.E.M., $\mathrm{n}=$ 7. Numbers on the $\mathrm{X}$-axis represent the dose in $\mathrm{mg} / \mathrm{kg} /$ day. Line above bar indicates significant differences $\mathrm{P}<0.05$

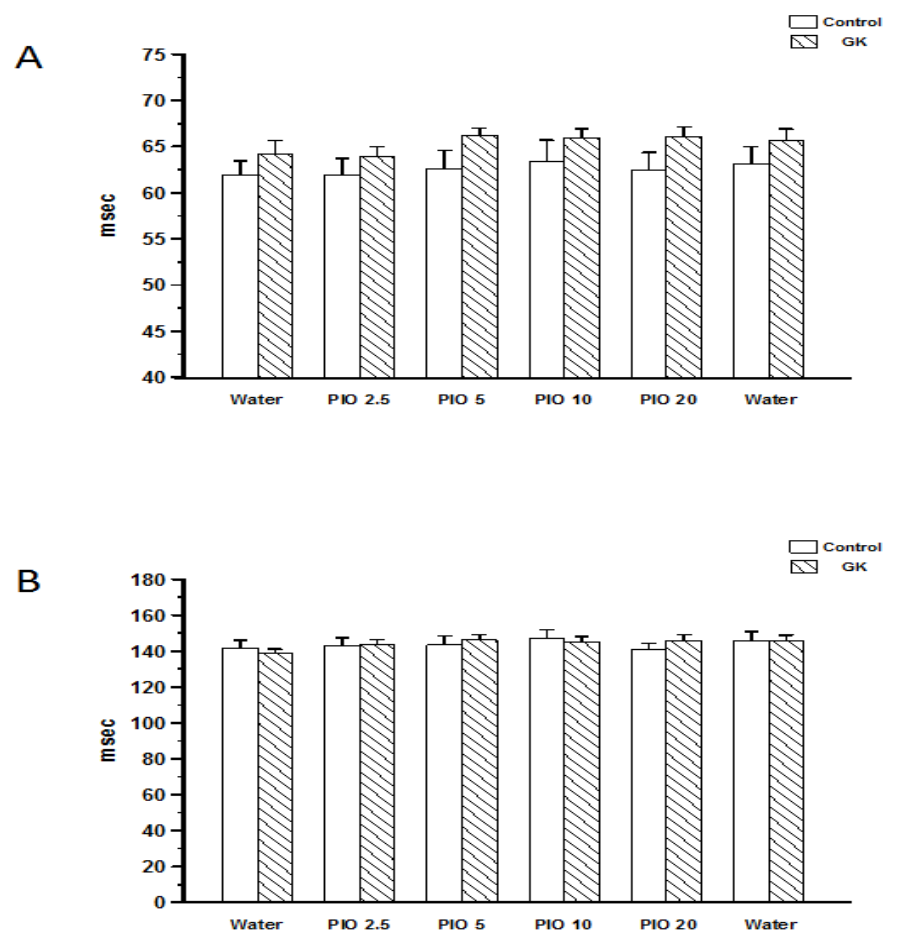

Figure 5: Effects of Pioglitazone (PIO) on QT and corrected QT interval in GK rats and controls. A: Mean QT interval and $B$ : Mean corrected QT interval in GK rats and controls during administration of different concentrations of PIO. Data are means \pm S.E.M., $\mathrm{n}=7$. Numbers on the $\mathrm{X}$-axis represent the dose in $\mathrm{mg} / \mathrm{kg} /$ day. 


\section{Discussion}

Pioglitazone is an exogenous thiazolidinedione and is used in the treatment of type 2 diabetes mellitus. PPAR- $\alpha$ and PPAR- $\gamma$ activator drugs variously improve dyslipidemia, insulin resistance and diabetes control. In clinical trials PIO appears to reduce cardiovascular events whilst rosiglitazone potentially increases the risk of myocardial infarction [21]. Some of the effects of PIO on lipid and glycemic metabolism, perhaps mediated by activation of PPAR- $\alpha / \gamma$, might have beneficial effects on the electrical conduction system of the diabetic heart. This study employed in vivo biotelemetry techniques to investigate the effects of PIO on the electrical conduction system of diabetic and age-matched control hearts. The main findings of the study were as a follows: (1) Bodyweight was higher in GK rats compared to controls and PIO treatment in the range 2.5 to $20 \mathrm{mg} / \mathrm{kg}$ did not significantly alter bodyweight in either GK rats or controls; (2) Non-fasting blood glucose was significantly elevated in GK rats compared to controls and with increasing concentrations of PIO treatment blood glucose in GK rats was progressively normalized towards control value; (3) Heart rate was modestly lower in GK rats compared to controls and was not altered by PIO; (4) PQ interval was prolonged in GK rats compared to controls and was not altered by PIO. (5) QRS complex duration and QT interval were not significantly different in GK rats compared to controls and were not altered by PIO.

Bodyweight was higher in GK rats compared to controls a finding that is consistent with some previous studies [18,19,22,23]. Thiazolidinediones are typically associated with weight gain and increased risk of edema [24] so it was interesting to note that $\mathrm{PIO}$, in the range of concentrations used in this study, had little effect on bodyweight in either GK rats or controls. Consistent with previous studies the non-fasting blood glucose was elevated in GK rats compared to controls [18,19,22] and treatment with increasing concentrations of PIO, blood glucose in GK rats was progressively improved towards control values.

Heart rate was modestly reduced in GK rats compared to controls and was not additionally altered by PIO. Previous in vivo biotelemetry studies have also reported reductions in heart rate in GK rats aged between 2 and 15 months compared to controls [19]. Reductions in heart rate might be attributed to altered autonomic control and/or altered intrinsic control of the heart. Action potential experiments in spontaneously beating isolated heart have shown reductions in heart rate suggesting that intrinsic mechanisms at least partly underlie the reduced heart rate in GK rats [23]. Alterations in sympathovagal control of the heart might also partly underlie the reduced heart rate in GK rats $[25,26]$. The PQ interval, time between atrial depolarization and the start of ventricular depolarization, was prolonged in GK rats compared to controls. This finding may suggest prolonged conduction time between the atria and ventricles which might be attributed to atrioventricular node dysfunction. Reduced heart rate and prolonged sinoatrial node conduction time have been previously demonstrated in the streptozotocin-induced diabetic rat heart [27]. The QRS complex, which represents ventricular depolarization, was not significantly reduced in GK rats compared to controls and was not additionally altered by PIO. Interestingly, previous studies have demonstrated prolonged QRS complex duration in GK rats compared to controls, however, the differences were only significant in young 2 month old rats and, consistent with the current study, were no longer significant in older animals aged 7 and 15 months [19]. Prolonged QT interval, which represents the time from ventricular depolarization to repolarization, is a frequently reported finding in diabetic patients [28]. In the current study QT interval and QT when corrected for heart rate were not altered in GK rats compared to controls and were not additionally altered by PIO. Previous studies have demonstrated small prolongations of QT interval in GK rats aged 2, 7 and 15 months [19].

Collectively, the data reported in this study have shown that bodyweight and blood glucose are elevated, heart rate is modestly reduced and PQ interval is prolonged in GK rats compared to Controls. PIO in the range 2.5-20 mg/kg administered orally during a period of 14 weeks had favorable effects on blood glucose and little or no effects on heart rate, QRS complex duration, QT and PQ intervals.

\section{Acknowledgment}

The project has been supported by grants from the College of Medicine \& Health Sciences and the United Arab Emirates University.

\section{References}

1. Malik M, Bakir A, Saab BA, King H (2005) Glucose intolerance and associated factors in the multi-ethnic population of the United Arab Emirates: results of a national survey. Diabetes Res Clin Pract 69: 188-95.

2. Clar C, Royle P, Waugh N (2009) Adding pioglitazone to insulin containing regimens in type 2 diabetes: systematic review and meta-analysis. PLoS One 4: e6112.

3. Derosa G (2010) Efficacy and tolerability of pioglitazone in patients with type 2 diabetes mellitus: comparison with other oral antihyperglycaemic agents. Drugs 70: 1945-61.

4. Grinsell JW, Lardinois CK, Swislocki A, Gonzalez R, Sare JS, et al. (2000) Pioglitazone attenuates basal and postprandial insulin concentrations and blood pressure in the spontaneously hypertensive rat. Am J Hypertens: 370-5.

5. Kemnitz JW, Elson DF, Roecker EB, Baum ST, Bergman RN, et al. (1994) Pioglitazone increases insulin sensitivity, reduces blood glucose, insulin, and lipid levels, and lowers blood pressure, in obese, insulin-resistant rhesus monkeys. Diabetes 43: 204-11.

6. Chinnam P, Mohsin M, Shafee LM (2012) Evaluation of acute toxicity of pioglitazone in mice. Toxicol Int 19: 250-4.

7. Hughes AD, Park C, March K, Coady E, Khir A, et al. (2013) A randomized placebo controlled double blind crossover study of pioglitazone on left ventricular diastolic function in type 2 diabetes. Int J Cardiol 167: 1329-32. 
8. van der Meer RW, Rijzewijk LJ, de Jong HW, Lamb HJ, Lubberink M, et al. (2009) Pioglitazone improves cardiac function and alters myocardial substrate metabolism without affecting cardiac triglyceride accumulation and high-energy phosphate metabolism in patients with well-controlled type 2 diabetes mellitus. Circulation 119: 2069-77.

9. Bakth S, Arena J, Lee W, Torres R, Haider B, et al. (1986) Arrhythmia susceptibility and myocardial composition in diabetes. Influence of physical conditioning. J Clin Invest 77: 382-95.

10. Ewing DJ, Boland O, Neilson JM, Cho CG, Clarke BF (1991) Autonomic neuropathy, QT interval lengthening, and unexpected deaths in male diabetic patients. Diabetologia 34: 182-5.

11. Lindstrom T, Jorfeldt L, Tegler L, Arnqvist HJ (1992) Hypoglycaemia and cardiac arrhythmias in patients with type 2 diabetes mellitus. Diabet Med 9: 536-41.

12. Movahed MR, Hashemzadeh M, Jamal MM (2005) Increased prevalence of third-degree atrioventricular block in patients with type II diabetes mellitus. Chest 128: 2611-4.

13. Stettler C, Bearth A, Allemann S, Zwahlen M, Zanchin L, et al. (2007) QTc interval and resting heart rate as long-term predictors of mortality in type 1 and type 2 diabetes mellitus: a 23-year follow-up. Diabetologia 50: 186-94.

14. Grimm W, Langenfeld H, Maisch B, Kochsiek K (1990) Symptoms, cardiovascular risk profile and spontaneous ECG in paced patients: a five-year follow-up study. Pacing Clin Electrophysiol 13: 2086-90.

15. Goto Y, Kakizaki M, Masaki N (1976) Production of spontaneous diabetic rats by repetition of selective breeding. Tohoku J Exp Med 119: 85-90.

16. Xu Z, Ju J, Wang K, Gu C, Feng Y (2014) Evaluation of hypoglycemic activity of total lignans from Fructus Arctii in the spontaneously diabetic Goto-Kakizaki rats. J Ethnopharmacol 151: 548-55.

17. Iltis I, Kober F, Desrois M, Dalmasso C, Lan C, et al. (2005) Defective myocardial blood flow and altered function of the left ventricle in type 2 diabetic rats: a noninvasive in vivo study using perfusion and cine magnetic resonance imaging. Invest Radiol 40: 19-26.

18. Salem KA, Adrian TE, Qureshi MA, Parekh K, Oz M, et al. (2012) Shortening and intracellular Ca2+ in ventricular myocytes and expression of genes encoding cardiac muscle proteins in early onset type 2 diabetic Goto-Kakizaki rats. Exp Physiol 97: 1281-91.

19. Howarth FC, Jacobson M, Shafiullah M, Adeghate E (2008) Long-term effects of type 2 diabetes mellitus on heart rhythm in the Goto-Kakizaki rat. Exp Physiol 93: $362-9$.

20. Howarth FC, Jacobson M, Shafiullah M, Adeghate E (2006) Effects of insulin treatment on heart rhythm, body temperature and physical activity in streptozotocin-induced diabetic rat. Clin Exp Pharmacol Physiol 33: 327-31.

21. Wilding JP (2012) PPAR agonists for the treatment of cardiovascular disease in patients with diabetes. Diabetes Obes Metab 14: $973-82$.

22. Salem KA, Qureshi MA, Sydorenko V, Parekh K, Jayaprakash P, et al. (2013) Effects of exercise training on excitation-contraction coupling and related mRNA expression in hearts of Goto-Kakizaki type 2 diabetic rats. Mol Cell Biochem 380: 83-96.

23. Howarth FC, Shafiullah M, Qureshi MA (2007) Chronic effects of type 2 diabetes mellitus on cardiac muscle contraction in the Goto-Kakizaki rat. Exp Physiol 92: 1029-36.

24. Hermansen K, Mortensen LS (2007) Bodyweight changes associated with antihyperglycaemic agents in type 2 diabetes mellitus. Drug Saf 30: $1127-42$.

25. Bootsma M, Swenne CA, Van Bolhuis HH, Chang PC, Cats VM, et al. (1994) Heart rate and heart rate variability as indexes of sympathovagal balance. Am J Physiol 266: H1565-71.

26. Ferrari AU, Daffonchio A, Albergati F, Mancia G (1991) Differential effects of aging on the heart rate and blood pressure influences of arterial baroreceptors in awake rats. J Hypertens 9: 615-21.

27. Howarth FC, Nowotny N, Zilahi E, El Haj MA, Lei M (2007) Altered expression of gap junction connexin proteins may partly underlie heart rhythm disturbances in the streptozotocin-induced diabetic rat heart. Mol Cell Biochem 305: 145-51.

28. Cox AJ, Azeem A, Yeboah J, Soliman EZ, Aggarwal SR, et al. (2014) Heart rate-corrected QT interval is an independent predictor of all-cause and cardiovascular mortality in individuals with type 2 diabetes: the Diabetes Heart Study. Diabetes Care 37: 1454-61.

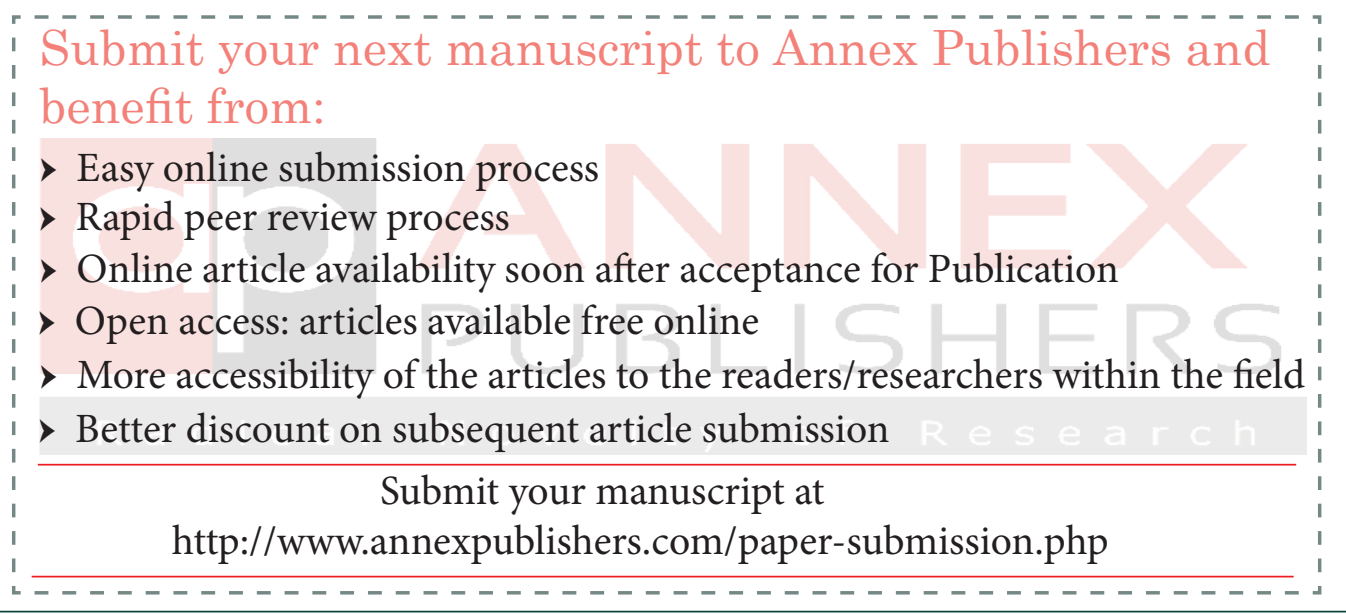

